\title{
Thermodynamics of the anisotropic two-channel Kondo problem
}

\author{
Gergely Zaránd ${ }^{1,2}$, Theo Costi $^{3}$, Andres Jerez ${ }^{3}$, and Natan Andrei ${ }^{4}$ \\ ${ }^{1}$ Lyman Physics Laboratory, Harvard University, Cambridge, $M A$ \\ ${ }^{2}$ Research Group of the Hungarian Academy of Sciences, Institute of Physics, TU Budapest, H-1521 \\ ${ }^{3}$ Institut Laue-Langevin, 6 rue Jules Horowitz, B.P. 156, 38042 Grenoble Cedex 9, France \\ ${ }^{4}$ Center of Materials Theory, Rutgers University, Piscataway, NJ 08854-0849, USA
}

(November 4, 2018)

\begin{abstract}
We construct and solve numerically the thermodynamic Bethe Ansatz equations for the spinanisotropic two-channel Kondo model in arbitrary external field $h$. At high temperatures the specific heat and the susceptibility show power law dependence. For $h \rightarrow 0$ and at temperatures below the Kondo temperature $T_{K}$ a two-channel Kondo effect develops characterized by a Wilson ratio $8 / 3$, and a logarithmic divergence of the susceptibility and the linear specific heat coefficient. Finite magnetic field, $h>0$ drives the system to a Fermi liquid fixed point with an unusual Wilson ratio which depends sensitively on $h$.

PACS numbers: 75.20.Hr, 71.10.Hf, 71.27.+a, 72.15.Qm
\end{abstract}

\section{INTRODUCTION}

The two-channel Kondo model (2CKM) attracted a lot of interest during the past few years. 1 This intense interest is mostly triggered by the rather unusual properties of this model: The existence of finite residual entropy in zero external field, the vanishing of single particle scattering amplitude at $T=0$ temperature, and the logarithmic singularity of various thermodynamic quantities. All these unusual properties appear due to the presence of a hidden and conserved flavor quantum number of the electrons.

Several physical systems have been proposed to realize the 2CKM. An unambiguous realization of the 2CKM is provided by dilute Uranium and Cerium-based heavy fermion compounds 1 In these alloys the combination of strong spin-orbit interaction and crystal field symmetry effects leads to an effective 2CKM.

It has been also suggested that non-commutative tunneling centers may form two-level systems (TLS) and realize the 2CKM. 2 In this case the localized spin is replaced by the position of the tunneling center, while the angular momenta of the conduction electrons play the role of the electron spin in the 2CKM. The electron spins in the TLS problem play the role of silent flavor indices. Though there have been several concerns raised concerning the microscopic structure of the TLS and the possibility of observing the two-channel Kondo behavior 3 many experiments may be consistently interpreted ip torms of such dynamical two-channel Kondo impurities. $\mathrm{E}$

Another realization of the $2 \mathrm{CKM}$ is provided hy quantum dots near to their charge degeneracy point. 1 In this case the charging states of the dot replace the impurity spin states and they couple to the position variable of the conduction electrons. Again, the spin of the electrons acts as a flavor variable. Though it appears to be extremely difficult to observe experimentally the nonFermi liquid beharior associated with the two-channel Kondo fixed point, 9 some fingerprints of the two-channel
Kondo effect have been observed in the capacitance of such semiconducting quantum dots 10

The above physical realizations of the 2CKM have the common feature that the 2CKM that describes them is in all cases generically strongly spin-anisotropic, and breaks the full $\mathrm{SU}(2)$ spin symmetry. While this $\mathrm{SU}(2)$ symmetry breaking is known to be irrelevant at the twochannel Kondo fixed point, 1 it affects both qualitatively and quantitatively the properties of the model at energies around and above the Kondo temperature, $T_{K}$, and has to be taken into account to make comparison with experiments.

In the present paper we generalize the Bethe Ansatz results of Ref. [11] to compute both analytically and numerically the thermodynamics of the anisotropic 2CKM at arbitrary temperatures and magnetic fields for various values of the anisotropy. Below the Kondo temperature, $T_{K}$, we find that the thermodynamic properties of the model are very similar to those of the isotropic 2 CKM. This observation is in full agreement with the irrelevance of the anisotropy at the 2CKM. However, above $T_{K}$ the behavior of the model depends crucially on spin anisotropy. We find that above $T_{K}$ all thermodynamic quantities display a power law behavior with an exponent determined by the value of the anisotropy. Furthermore, we find, that at finite magnetic fields the system flows to an unusual Fermi liquid fixed point with an anisotropydependent Wilson ratio.

The paper is organized as follows. In Section II we introduce the 2CKM. In Section III] we construct the thermodynamic Bethe Ansatz equations for the anisotropic $2 \mathrm{CKM}$ that we analyze in detail in Sec. IV. Some details of the computation are given in the Appendix.

\section{MODEL}

The anisotropic 2 CKM consists of a spin $S_{0}=1 / 2 \mathrm{im}-$ purity that couples dynamically to the spin of the conduction electrons through a strongly anisotropic exchange 
interaction, and is described by the following first quantized Hamiltonian $\left(\hbar=k_{B}=\mu_{B}=1\right)$ :

$$
\begin{aligned}
H & =\sum_{j=1}^{N_{e}}\left\{-i \partial / \partial x_{j}+\sum_{\alpha=x, y, z} \delta\left(x_{j}\right) J_{\alpha} S_{j}^{\alpha} S_{0}^{\alpha}\right\} \\
& +h\left(g S_{0}^{z}+g^{\prime} \sum_{j} S_{j}^{z}\right) .
\end{aligned}
$$

In this equation $x_{j}$ is the coordinate of the $j$ 'th conduction electron, $N_{e}=N \times f$ is the total number of electrons, and the $S_{j}$ 's $\left(j=0, . ., N_{e}\right)$ denote the spin $1 / 2$ operators of the electrons. Electrons also have a conserved flavor quantum number, $m=\{1, . ., f\}$, implicit in Eq. (11). In the following we discuss the case $f \geq 2$ as well though for the physical realizations mentioned in the Introduction $f=2$. (The $f=1$ case has been analyzed in Refs. [ 12, 11.)

The meaning of the various terms in this Hamiltonian depends on the particular physical realization. The external field $h$ couples to the local moment and the conduction electron spins with different g-factors. In the TLS problem and the quantum dot case, the external field $h$ represents the asymmetry of the TLS and the splitting between the two charging states of the quantum dot, respectively, and $g^{\prime} \equiv 0$. For heavy fermion systems, depending on the particular realization, $h$ can describe an external magnetic- or strain field, and may also couple to $\sum_{j} S_{j}^{z}$. In the present paper we mostly study the local susceptibility, corresponding to $g^{\prime}=0$.

In the original formulation of the Bethe Ansatz, similar to the conformal field theory solution of the Kondo problem 13 the impurity spin is 'fused' with the spin degrees of freedom of the conduction electrons, and the external field couples to the total spin, corresponding to $g^{\prime}=1$. Thus the Bethe Ansatz calculates the impurity contribution to the global susceptibility with $g^{\prime}=g$. Lowenstein made a remarkable attempt to treat the $g^{\prime} \neq$ 1 case, 14 however, his results were not fully conclusive. 14 In the present paper we use a different strategy to cope with the $g^{\prime}=0$ situation, and show that, similar to the case of the single channel anisotropic Kondo problem,t5 the $g^{\prime}=0$ local susceptibility (i.e. the susceptibility arising when the field couples only to the impurity spin) is simply proportional to the global susceptibility. Therefore, after determining the appropriate normalization factor, we are able to extract the exact local susceptibility from the Bethe Ansatz calculation with $g=g^{\prime}$.

In our analysis we shall assume that $J_{z} \neq J_{x}=J_{y}=$ $J_{\perp}$ and thus the Hamiltonian has a $U(1)$ spin symmetry. This is true for the quantum dot and heavy fermion realizations, however, for the TLS problem $J_{z} \gg J_{x}>$ $J_{y}=0$ Fortunately, it is not necessary to treat the general Bethe Ansatz equations in order to determine the universal features of the TLS Hamiltonian in the TLS case either: Under scaling the terms $J_{x}$ and $J_{y}$, describing electron-assisted tunneling processes, become rapidly equall and therefore it suffices to consider an equivalent effective model with $J_{x}^{\text {eff }}=J_{y}^{\text {eff }}=J_{\perp}=J_{x} / 2$ to determine the thermodynamics. This effective model has $U(1)$ orbital symmetry and can be much more easily analyzed by Bethe Ansatz techniques than the fully anisotropic one.

In constructing the wave function we implicitly have to introduce a further electron-electron interaction

$$
H^{e l-e l}=\sum_{1 \leq i<j \leq N_{e}} \sum_{\alpha=x, y, z} \delta\left(x_{i}-x_{j}\right) J_{\alpha} S_{i}^{\alpha} S_{j}^{\alpha} .
$$

Since electrons move with the same velocity and in the same direction, this interaction does not modify the thermodynamics in a crucial way for $J_{z}>J_{\perp}$. In particular, we have shown that the specific heat of the electrons is unaffected by this interaction. However, it does rescale the g-factor in Eq. (11): $g \rightarrow g^{\text {eff }}$. Fortunately, we shall be able to compensate this effect by the renormalization of $g$-factors in the magnetic field term.

For $J_{z}<J_{\perp}$ the artificial electron-electron interaction apparently generates a gap in the spin sector, and therefore it is impossible to capture the Kondo effect there within the algebraic Bethe Ansatz approach. Therefore, unfortunately, we have to restrict our discussion to the case $J_{z}>J_{\perp}$, and our calculations are not directly applicable to the quantum dot case.

In Eq. 1, only a term $\sim h S_{0}^{z}$ has been included, which acts as a local field in the $z$ direction. In reality, however, the field can also point into the 'perpendicular' direction corresponding to a term $\sim h_{\perp} S_{0}^{x}$. In the TLS case this term describes spontaneous tunneling between the TLS positions. The general effect of the latter term is very similar to that of $h S_{0}^{z}$, but quantitatively it behaves somewhat differently. For a TLS, e.g., in a typical situation $h$ is believed to be much larger than $h_{\perp}, \underline{W}$ and furthermore, its effective value is orders of magnitude reduced by polaronic effects. 227 Therefore, in most situations it is enough to include only the term $\sim h S_{0}^{z}$ in the Hamiltonian.

\section{BETHE-ANSATZ SOLUTION}

The most important ingredients of the algebraic Bethe Ansatz (BA) are the various scattering matrices. The impurity-conduction electron S-matrix can be constructed directly from (11):

$$
R_{0 j}=U_{0 j}^{(s p i n)}\left(\lambda_{0}-\lambda_{j}\right)_{\sigma_{0} \sigma_{0}^{\prime} ; \sigma_{j} \sigma_{j}^{\prime}} \otimes I d^{(\text {flavor })},
$$

where $\lambda_{0}=-1$ and $\lambda_{j}=\rightarrow 0$ denote the "rapidities" of the impurity and conduction electron $j$, and $U$ is the $U(1)$ scattering matrix

$$
\begin{aligned}
U_{0 j}(\lambda) & =a(\lambda) P_{\uparrow \uparrow}+b(\lambda) P_{\uparrow \downarrow}+\frac{1}{2} c(\lambda)\left(S_{0}^{+} S_{j}^{-}+S_{0}^{-} S_{j}^{+}\right), \\
\frac{a(\lambda)}{c(\lambda)} & =\frac{\sinh (i \mu+\lambda \vartheta)}{\sinh (i \mu)}, \quad \frac{a(\lambda)}{b(\lambda)}=\frac{\sinh (i \mu+\lambda \vartheta)}{\sinh (\lambda \vartheta)},
\end{aligned}
$$


with $P_{\uparrow \uparrow}$ and $P_{\uparrow \downarrow}$ being the projection operators for parallel and opposite spins. Excepting the small coupling regime, the connection of the parameters 4 and $\vartheta$ with the bare couplings $J_{z}$ and $J_{\perp}$ is ambiguous 12 16 and depends on the regularization procedure of the Dirac delta and the cutoff scheme used. Therefore, instead of $J_{z}$ and $J_{\perp}$, it is rather $\mu$ and $\theta$ that should be viewed as the basic parameters of the BA solution: $\mu$ turns out to be connected to the renormalized phase shift while the ratio $\mu / \theta$ determines the Kondo temperature, below which non-Fermi-liquid correlations appear:

$$
T_{K}=\left(1-f \frac{\mu}{\pi}\right) 2 D \exp \{-\pi \theta / \mu\}
$$

with $D=N / L$.

Since electrons move with the same velocity we have the liberty to define their scattering matrix in a way to maintain integrability:

$$
R_{i j}=U_{i j}\left(\lambda_{i}-\lambda_{j}\right) \otimes F_{i j}\left(\lambda_{i}-\lambda_{j}\right) .
$$

Here $U(\lambda)$ is given by Eq. (4) and $F$ describes scattering in the flavor sector:

$$
F_{i j}\left(\lambda_{i}-\lambda_{j}\right)=\frac{\lambda_{i}-\lambda_{j}+i c X_{i j}}{\lambda_{i}-\lambda_{j}+i c},
$$

with $X_{i j}$ the flavor exchange operator of particle $i$ and $j$ and $c$ an arbitrary constant to be defined later.

Starting from these scattering matrices we used the algebraic BA to determine the nested BA equations and then applied the dynamical fusion procedure of Ref. [17] to eliminate the flavor degrees of freedom. The fused equations considerably simplify with the choice $c \equiv \frac{\mu}{\vartheta}$. Then the rapidities $\left\{\lambda_{\alpha} ; \alpha=1, . ., M\right\}$ describing the spin sector of the wave function satisfy

$$
\begin{array}{r}
\frac{\sinh \left(\mu\left(\lambda_{\alpha}+\frac{i}{2}\right)+\vartheta\right)}{\sinh \left(\mu\left(\lambda_{\alpha}-\frac{i}{2}\right)+\vartheta\right)}\left[\frac{\sinh \mu\left(\lambda_{\alpha}+i \frac{f}{2}\right)}{\sinh \mu\left(\lambda_{\alpha}-i \frac{f}{2}\right)}\right]^{N}= \\
-\prod_{\beta=1}^{M} \frac{\sinh \mu\left(\lambda_{\alpha}-\lambda_{\beta}+i\right)}{\sinh \mu\left(\lambda_{\alpha}-\lambda_{\beta}-i\right)} .
\end{array}
$$

The momenta of the electrons and thus the total energy is determined by the periodic boundary conditions

$$
e^{i k_{A} L f}=\prod_{\alpha=1}^{M} \frac{\sinh \mu\left(\lambda_{\alpha}+i \frac{f}{2}\right)}{\sinh \mu\left(\lambda_{\alpha}-i \frac{f}{2}\right)} ; \quad E=\sum_{A=1}^{N} f k_{A},
$$

where $f k_{A}$ denotes the total momentum of the fused $f$ electron composites, and $L$ is the system size.

In the thermodynamic limit, $L, N \rightarrow \infty, N / L=$ $D$, the 'spin rapidities' $\lambda_{\alpha}$ in Eq. (8) are organized into strings 1211 of length $r$ and parity $v= \pm: \lambda \rightarrow$ $\left\{\lambda_{q}^{(r, v)} ; q=1, . ., r\right\}$ with

$$
\lambda^{(r, v)} \leftrightarrow \lambda_{q}^{(r, v)}=\lambda^{(r, v)}+\left[\frac{r+1}{2}-q\right]+i \frac{\pi}{4 \mu}(1-v) .
$$

We have verified that to obtain a stable solution for $\mu<$ $\pi / f, v$ and $r$ must satisfy the same stability condition as for $f=1,12$

$$
v \sin (\mu q) \sin (\mu(r-q))>0, \quad q=1, . .,[r / 2] .
$$

As shown by Takahashi and Susuki, 18 the allowed $(r, v)$ strings can be classified on the basis of an infinite (or finite) fraction expansion of $\mu / \pi$. To be specific, here we only discuss the simplest case $\mu=\pi / \nu$ and $f<\nu$, where only $\nu$ different stable string configurations exist: $n=(r, v)=(1,+),(2,+), . .,(\nu-1,+)$ and $(1,-)$. The case $\mu=\pi / f$ represents a singular limit: 19 For $f=1$ it corresponds to the decoupling point 20 while for $f=2$ it can be identified with the Emery-Kivelson point (see below).

\section{THERMODYNAMICS}

\section{A. Thermodynamic Bethe-Ansatz equations}

To derive the thermodynamic BA equations in the continuum limit $L, N \rightarrow \infty$ and $D \equiv N / L=$ cst we proceeded in the usual way. We first defined the density of rapidities (rapidity holes), $\varrho_{n}(\lambda)\left(\tilde{\varrho}_{n}(\lambda)\right)$. These are related to the 'excitation energies' $\epsilon_{n}(\lambda)$ through $\eta_{n} \equiv \tilde{\varrho}_{n} / \varrho_{n} \equiv e^{\varepsilon_{n} / T}$. The functions $\epsilon_{n}(\lambda)$ are determined by the following integral equations for $\nu>f$ :

$$
\begin{aligned}
\varepsilon_{\nu} / T & =g h \nu / 2 T-s * \ln \left(1+e^{\varepsilon_{\nu-2} / T}\right)+\delta_{\nu, f+1} \Theta(\lambda) \\
\varepsilon_{\nu-1} / T & =g h \nu / 2 T+s * \ln \left(1+e^{\varepsilon_{\nu-2} / T}\right)-\delta_{\nu, f+1} \Theta(\lambda) \\
\varepsilon_{j} / T & =s * \ln \left[\left(1+e^{\varepsilon_{j+1} / T}\right)\left(1+e^{\varepsilon_{j-1} / T}\right)\right] \\
& +\delta_{j, \nu-2} s *\left(1+e^{-\varepsilon_{\nu} / T}\right)-\delta_{j, f} \Theta(\lambda) \quad(j<\nu-1) .
\end{aligned}
$$

where $s *$ denotes convolution with the Kernel $s(\lambda)=$ $1 / \cosh (\pi \lambda)$, the driving term is given by $\Theta(\lambda)=$ $2 D / T \operatorname{arctg}\left(e^{\pi \lambda}\right)$ and $\varepsilon_{0} \rightarrow-\infty$. The impurity contribution to the free energy is given by

$$
F^{\mathrm{imp}}=-T \int_{-\infty}^{\infty} s\left(\lambda+\frac{\mu}{\vartheta}\right) \ln \left(1+\exp \left(\varepsilon_{1}(\lambda) / T\right)\right) d \lambda,
$$

and, in principle, all thermodynamic quantities can be calculated by taking the derivatives of $F^{\mathrm{imp}}$.

\section{B. Analytical results}

Many of the thermodynamic properties can be determined from the asymptotic form of the $\eta_{n}$ 's. Using the Ansatz $\eta_{n}(\lambda \rightarrow \pm \infty) \approx \eta_{n}^{ \pm}+b_{n}^{ \pm} e^{\mp \pi \tau_{ \pm} \lambda}$ one obtains a set of algebraic equations for the $\eta_{n}^{ \pm}$'s, $b_{n}^{ \pm}$'s and the exponents $\tau_{ \pm}$. The latter exponents govern the scaling of the free energy in the vicinity of the low- and highenergy fixed points and are given by $\tau_{+}=4 /(2+f)$ and 
$\tau_{-}=2 \mu / \pi$. The crossover between the two regimes occurs at the Kondo scale Eq. (5), which emerges naturally if one rewrites the thermodynamic BA equations abow in a 'universal' form by removing the cutoff $D .21,19,22$ The asymptotic form of the impurity free energy for $h \ll T \ll T_{K}$ is given by

$$
-F^{\mathrm{imp}} / T \sim \begin{cases}S^{\mathrm{imp}}+\left(a+b\left(\frac{g h}{T}\right)^{2}\right)\left(\frac{T}{T_{K}}\right)^{4 / 2+f} & f>2, \\ S^{\mathrm{imp}}+\left(a+b\left(\frac{g h}{T}\right)^{2}\right)\left(\frac{T}{T_{K}} \ln \left(\frac{T}{T_{K}}\right)\right) & f=2,\end{cases}
$$

implying the divergence of the linear specific heat coefficient $c / T$ at $h=0$ and the susceptibility as $T \rightarrow 0$. The constants $a$ and $b$ above depend on the specific value of $f$ and $\mu$, and the residual entropy $S^{\mathrm{imp}}$ is the same as in the isotropic case 17

$$
S^{\mathrm{imp}}=\ln \left[\frac{\sin \left(\frac{f \pi}{f+2}\right)}{\sin \left(\frac{\pi}{f+2}\right)}\right] .
$$

To determine the renormalization of the $g$-factor and the Wilson ratio, we calculated the linear specific heat coefficient and the bulk magnetization $M_{z}^{\text {tot }} \equiv-\partial F^{\text {tot }} / \partial h$ in the absence of the impurity spin (but with $g^{\prime}=g$ ). Similar to the case $f=1,12$ the linear specific heat coefficient agrees with that of the spin sector of non-interacting free electrons. However, the magnetization does not. Following similar lines as in Ref. [12, the total spin can be related to $\eta_{\nu}^{ \pm}$and $\eta_{\nu-1}^{ \pm}$and thus the magnetization is simply given by

$$
M^{z}=-\frac{\partial}{\partial h} F^{\mathrm{tot}}=g\left\langle\sum_{i=1}^{N_{e}} S_{i}^{z}\right\rangle=g^{2} \frac{f L}{4 \pi} \frac{h}{1-f \mu / \pi},
$$

with the term, $g^{2} f L / 4 \pi$, the Pauli susceptibility of free electrons.

From this equation it immediately follows by integration that at zero temperature

$$
F^{\mathrm{tot}}=-\frac{1}{2} g^{2} \frac{f L}{4 \pi} \frac{h^{2}}{1-f \mu / \pi} .
$$

Thus the g-factor is renormalized due to the electronelectron interaction as $g \rightarrow g /(1-\mu f / \pi)^{1 / 2}$, and to compensate the effect of Eq. (2), we have to chose

$$
g \equiv(1-\mu f / \pi)^{1 / 2} .
$$

Having thus compensated the effect of the artificial electron-electron interaction of Eq. (2) by rescaling $g$, we can proceed to calculate the impurity contribution to the global susceptibility (defined with $g^{\prime}=1, g=1$ but no electron-electron interaction). We find indeed that with the choice $g \equiv(1-\mu f / \pi)^{1 / 2}$ the low temperature (global) Wilson ratio, defined in terms of this global impurity susceptibility, takes on a universal value

$$
R_{\mathrm{glob}}^{\mathrm{imp}}=\lim _{T \rightarrow 0} \lim _{h \rightarrow 0} \frac{c^{\mathrm{bulk}}}{\chi^{\text {bulk }}} \frac{\chi_{\text {glob }}^{\text {imp }}}{c_{\text {glob }}^{\text {imp }}}=\frac{8}{3},
$$

as in the isotropic case, 23 proving again that exchange anisotropy is irrelevant at the two-channel Kondo fixed point 2426 In the following we shall always denote quantities that were calculated without (i.e. compensating) the artificial electron-electron interaction by the superscript 'imp'.

To capture the meaning of the parameter $\mu$ we also determined the impurity contribution to the global susceptibility in the high temperature regime:

$$
\chi_{\mathrm{glob}}^{\mathrm{imp}}=\frac{g^{2}}{4 T}=\frac{1-f \mu / \pi}{4 T} .
$$

Using Abelian bosonization techniques we were also able to prove analytically that for $J_{\perp} \ll J_{z}$ at high temperatures

$$
\chi_{\text {glob }}^{\text {imp }}(T \rightarrow \infty)=\left(1-2 f \frac{\delta}{\pi}\right)^{2} \frac{1}{4 T},
$$

with $\delta$ the phase shift generated by $J_{z}$ (see Appendix A for the-ferivation and the precise definition of the phase shift).27.25

This immediately implies the important relation

$$
\frac{\mu}{\pi}=4 \frac{\delta}{\pi}-4 f \frac{\delta^{2}}{\pi^{2}}
$$

Comparing this expression with the results of Ref. [27] we notice immediately that $\mu / \pi$ is nothing but the scaling dimension of $J_{\perp}$, which satisfies the folloving scaling equation at energy scales $\omega$ well above $T_{K}$ :27

$$
\frac{d \ln J_{\perp}}{d \ln \left(\omega_{0} / \omega\right)}=\frac{\mu}{\pi}, \quad\left(\omega \gg T_{K}\right) .
$$

Here $\omega_{0}$ is a high energy cutoff. For a TLS it is of the order of the Debye temperature, while for heavy fermions it is usually of the order of the Fermi energy. For a quantum dot the cutoff is the charging energy, $\omega_{0} \sim E_{c}$. The effective perpendicular coupling $J_{\perp}$ at energy scale $\omega$ can be obtained by simply integrating this equation.

Eq. (18) is further confirmed by noticing that for $f=2$ at the Emery-Kivelson line, $\delta=\pi / 4$, the global susceptibility Eq. (16) identically vanishes, in complete agreement with the results of Refs. [28]. The point $\delta=\pi / 2 f$ corresponding to $\mu=\pi / f$ is highly singular, and needs special care 19 At this particular point the amplitude of the leading irrelevant operator, responsible for the divergence of the susceptibilify and the linear specific heat coefficient, becomes zero.28

The global susceptibility, $\chi_{\text {glob }}^{\mathrm{imp}}$, and the associated global Wilson ratio, $R_{\text {glob }}^{\mathrm{imp}}$, defined in Eq. 15, are useful for magnetic Kondo systems. However, for quantum dots and TLS's it is rather the local impurity susceptibility that is of interest, i.e., the response of the system to an external field coupled only to the impurity spin. Hence, we studied the impurity contribution to the susceptibility when $g=1$ and $g^{\prime}=0$ (and in the absence of the artificial electron-electron interaction). In order to determine 
this we generalized the path integral derivation of Ref. [ 15] to show that

$$
F^{\mathrm{imp}}\left(h, T, g, g^{\prime}=g\right)=F^{\mathrm{imp}}\left(\tilde{h}, T, g, g^{\prime}=0\right),
$$

where $\tilde{h}=h(1-2 f \delta / \pi)=h(1-\mu f / \pi)^{1 / 2}$. To establish the second equality we used Eq. (18). Thus we can calculate the local impurity properties (those for $g=1, g^{\prime}=0$ and no electron-electron interaction) from the BA equations with $g^{\prime}=g=(1-f \mu / \pi)^{1 / 2}$ by simply rescaling the field $h$ according to Eq. (20).20

Henceforth, unless otherwise stated, we shall denote the local impurity susceptibility obtained from the BA solution in combination with Eq. (20) by $\chi^{\mathrm{imp}}$ and the associated local Wilson ratio by $R^{\text {imp }}$ (see below).

Following the above procedure we find at high temperature

$$
\begin{aligned}
\chi^{\mathrm{imp}} & =\frac{1}{4 T}\left[1-B\left(\frac{T_{K}}{T}\right)^{2 \mu / \pi}\right], \\
C^{\mathrm{imp}} & \sim\left(\frac{T_{K}}{T}\right)^{2 \mu / \pi} .
\end{aligned}
$$

Note that at high temperatures the specific heat exhibits a power law behavior which crosses over to a logarithmic behavior in the isotropic case $\mu \rightarrow 0$. Similarly, the corrections to the susceptibility about the free behavior are power law like and these power laws give way to logarithmic corrections in the isotropic limit $\mu \rightarrow 0$. We note that the above exponent $2 \mu / \pi$ is formally the same as found for the $f=1$ case 11 . However, the relation between $\mu$ and the bare couplings is quite different in the two cases and involves the channel number $f$ (see Eq. (18)).

The power-law corrections can be very easily understood from the scaling picture. Expanding the free energy in terms of $J_{\perp}$ one finds that the leading correction is second order in $J_{\perp}$. Making use of the scaling equation Eq. (19) it immediately follows that the leading corrections to the free energy behave as $T^{1-2 \mu / \pi}$, implying Eq. (22). Similar arguments lead to the conclusion that the impurity-induced resistivity correction behaves at high temperatures as

$$
\rho^{\mathrm{imp}}=A+B\left(\frac{T_{K}}{T}\right)^{2 \mu / \pi}
$$

for $T \gg T_{K}$.

At low temperatures, $T \ll T_{K}$, some care is required in discussing thermodynamic properties. In contrast the case $f=1$, where $F$ is analytical around $T=h=012$ here the $h=0, T=0$ point is an essential singularity and the two limits $T \rightarrow 0$ and $h \rightarrow 0$ are not interchangeable. Taking the limit $h \rightarrow 0$ first, we find the following nonFermi liquid behavior

$$
\begin{gathered}
\chi^{\mathrm{imp}} \sim \frac{1}{T_{K}} \ln \left(\frac{T}{T_{K}}\right), \\
C^{\mathrm{imp}} \sim \frac{T}{T_{K}} \ln \left(\frac{T}{T_{K}}\right),
\end{gathered}
$$

$$
\tilde{R}^{\mathrm{imp}}=\lim _{T \rightarrow 0} \lim _{h \rightarrow 0} \frac{c^{\mathrm{bulk}}}{\chi^{\mathrm{bulk}}} \frac{\chi^{\mathrm{imp}}}{C^{\mathrm{imp}}}=\frac{1}{1-f \mu / \pi} \frac{8}{3} .
$$

Here, we have defined a local Wilson ratio, $\tilde{R}^{\text {imp }}$. It is expressed in terms of the local impurity susceptibility $\chi^{\text {imp }}$, and the impurity contribution to the specific heat $c^{\mathrm{imp}}$. It differs from the Wilson ratio defined in Eq. 15 (which is the usual definition for this quantity in the magnetic Kondo problem) by having $g^{\prime}=0$. We see that this local Wilson ratio depends on anisotropy $\mu$ and thus the phase shift $\delta$, and is therefore not universal.

We now consider the limit of $T \rightarrow 0$, with $h$ remaining either finite, or taken to zero subsequently. In this case, and for $h \ll T_{K}$, we find from the numerical results of the next section, that there is a low energy scale $T_{\mathrm{FL}}=$ $h^{2} / T_{K}$, below which the thermodynamics is Fermi liquid like. In particular for $T \ll T_{\mathrm{FL}}$ and small magnetic fields $h \ll T_{K}$ we find

$$
\begin{aligned}
& \chi^{\mathrm{imp}}\left(h, T \ll T_{\mathrm{FL}}\right) \sim-\ln \left(h / T_{K}\right), \\
& C^{\mathrm{imp}}\left(h, T \ll T_{\mathrm{FL}}\right) \sim \frac{T}{T_{F}},
\end{aligned}
$$

with $T_{\mathrm{FL}} \sim h^{2} / T_{K}$ (for $h \ll T_{K}$ ). A local Wilson ratio for arbitrary local magnetic field $h$ can be defined as

$$
R^{\mathrm{imp}}(h)=\lim _{T \rightarrow 0} \frac{c^{\mathrm{bulk}}(h)}{\chi^{\mathrm{bulk}}(h)} \frac{\chi^{\mathrm{imp}}(h)}{C^{\mathrm{imp}}(h)} .
$$

In contrast to the $f=1$ case, for which this quantity is independent of $h$, but dependent on-anisotropy (being given by $R^{\mathrm{imp}, \mathrm{f}=1}(h)=2 /(1-\mu / \pi) 11$, 11$)$, for the present $f=2$ case it depends explicitly on both $h$ and anisotropy (Fig. 6). This important result, which is consistent with the result for the isotropic case 32 , will be discussed in the following section on the numerical solution. We note here, however, that the local Wilson ratio for the $f=2$ case agrees with the $f=1$ local Wilson ratio in the case of asymptotically large magnetic fields, $h \gg T_{K}$, i.e. in this case we have

$$
R^{\mathrm{imp}}\left(h \gg T_{K}\right)=\frac{2}{1-\frac{\mu}{\pi}},
$$

although the meaning of $\mu$ is different in the two cases (see Eq. (18)). The detailed dependence of this local Wilson ratio on $h$ will be discussed in the next section.

\section{Numerical solution}

In order to obtain the thermodynamics at all temperatures, it was necessary to solve the thermodynamic BA equations of Sec.IVA numerically. A procedure for doing this, which is valid for arbitrary values of the magnetic field $h$ and temperature has been developed in Ref. [ 11] for $f=1$. With small modifications, the same procedure applies also to the present case. We considered 


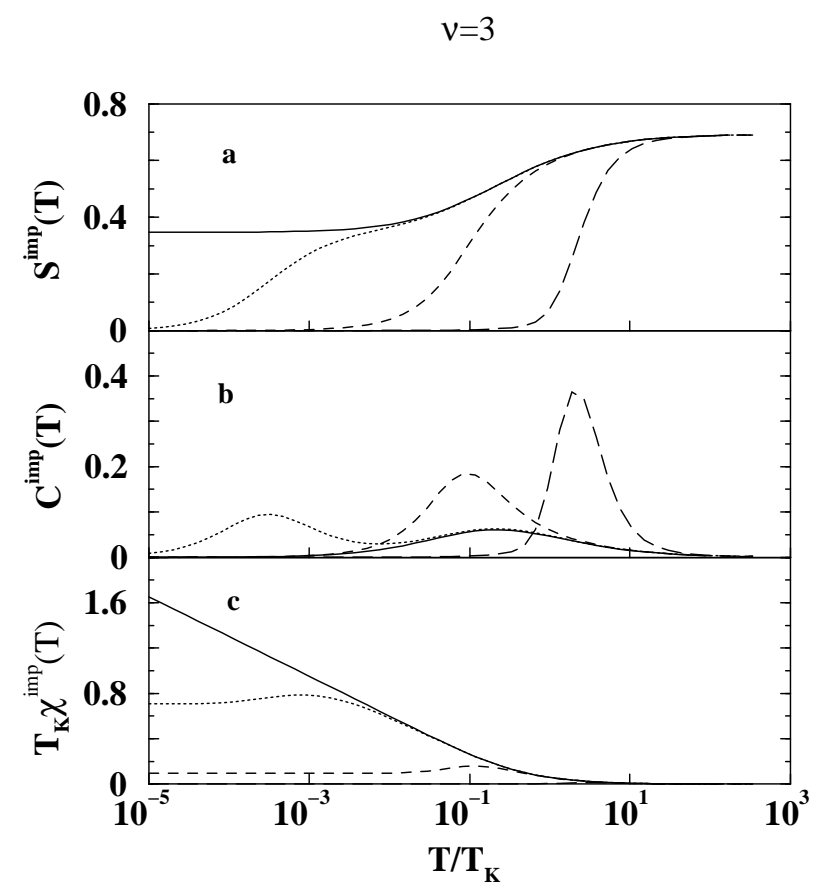

FIG. 1. The impurity contribution to the entropy, $S^{\mathrm{imp}}$, specific heat, $C^{\text {imp }}$, and (local) susceptibility, $\chi^{\text {imp }}$, as functions of $T / T_{K}$ for magnetic fields $h / T_{K}=2^{-6}$ (solid), $h / T_{K}=2^{-4}$ (dotted), $h / T_{K}=1$ (dashed) and $h / T_{K}=2^{4}$ (long-dashed) for the case $\nu=3$ corresponding to the largest anisotropy studied. $\mathrm{h} / \mathrm{T}_{\mathrm{K}}=2^{-4}$

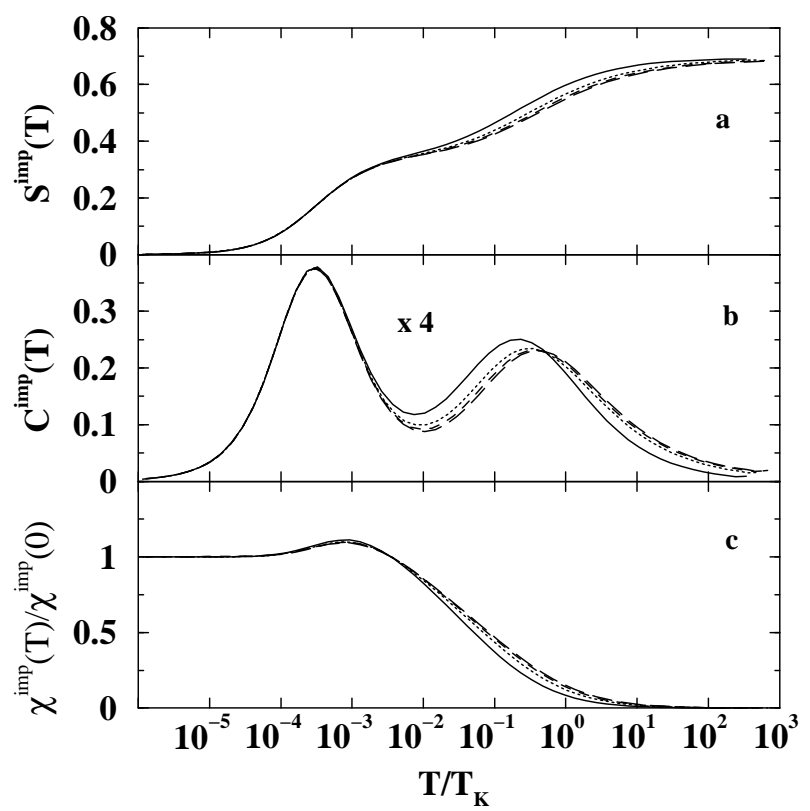

FIG. 2. The anisotropy dependence of the entropy, specific heat and susceptibility at small magnetic fields $\left(h / T_{K}=2^{-4}\right)$, (note that (b) is scaled by a factor 4 for comparison with the corresponding case of large magnetic fields shown in Fig. 3). The anisotropies shown are for $\mu / \pi=1 / \nu$ with $\nu=3$ (solid), $\nu=4$ (dotted), $\nu=5$ (dashed) and $\nu=6$ (long-dashed)

anisotropies given by $\mu / \pi=1 / \nu$ with $\nu=3,4,5,6$. In Fig. 11a-c we show the thermodynamics of the anisotropic 2CKM for a large anisotropy $(\nu=3)$ as one might have in a realistic system. The characteristic non-Ermi liquid behavior, in particular the $\ln (2) / 2$ entropy 17 and the logarithmically divergent $\chi^{\mathrm{imp}}(T)$ and $C^{\mathrm{imp}}(T) / T$, are found at zero field. A finite field, $h>0$, restores Fermi liquid behavior at temperatures below a low energy scale $T_{\mathrm{FL}}=h^{2} / T_{K}$, as found for the isotropic, $\nu=\infty$, case 32 . The non-Fermi liquid behavior for $0<h<T_{K}$ is therefore restricted to an intermediate range of temperatures, $T_{\mathrm{FL}}<T<T_{K}$, and we see that a clear signature of such behavior (such as the two peaks in the specific heat with each peak having only $\ln (2) / 2$ entropy, or a $\ln (T)$ behavior of $\chi(T)$ for a temperature range below $\left.T_{K}\right)$ is possible, even at moderate magnetic fields, $h \sim T_{K} / 16$.

In Fig. 2a-c and Fig. 3a-c we show the effect of different anisotropies on the thermodynamics for both a small external fields $\left(h \ll T_{K}\right.$, Fig. 2) and a large magnetic fields $\left(h \gg T_{K}\right.$, Fig. 3). At $h \ll T_{K}$, the main effect of anisotropy is to modify the thermodynamics at intermediate, $T_{\mathrm{FL}} \lesssim T \lesssim T_{K}$, and high temperatures, $T>T_{K}$. For large magnetic field, $h \gg T_{K}$, the anisotropy modifies the thermodynamic properties at temperatures, $T \gtrsim T_{K}$ (Fig. 3).

A characteristic feature of the present model $(f=2)$, is 


$$
\mathrm{h} / \mathrm{T}_{\mathrm{K}}=2^{4}
$$

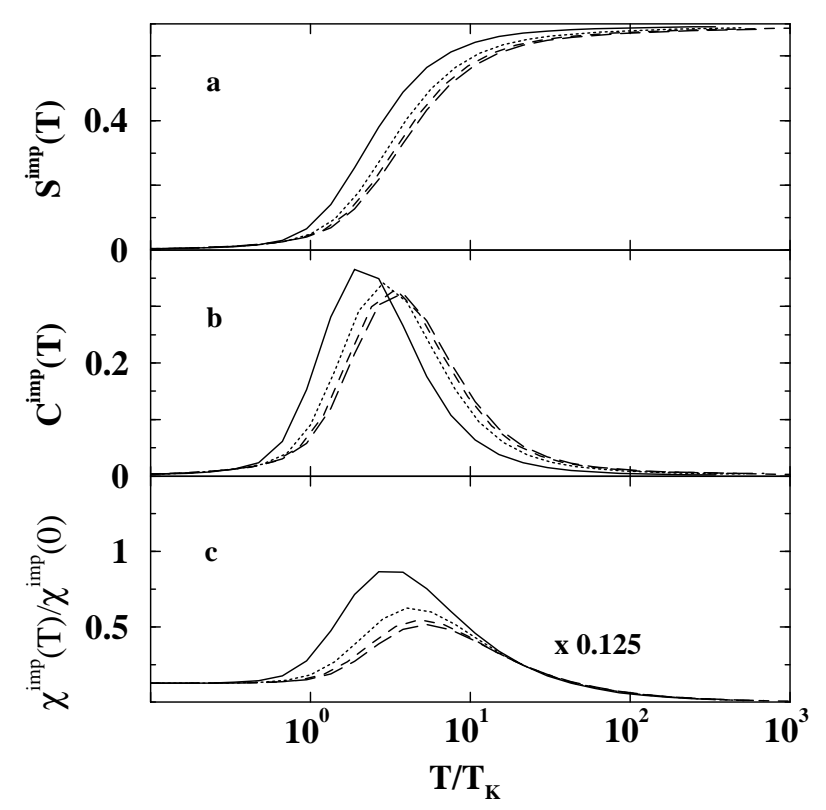

FIG. 3. The anisotropy dependence of the entropy, specific heat and susceptibility at large magnetic fields $\left(h / T_{K}=2^{4}\right)$, (note that (c) is scaled by a factor 0.125 for comparison with the corresponding case for small magnetic fields shown in Fig. 2 ). The anisotropies shown are for $\mu / \pi=1 / \nu$ with $\nu=3$ (solid), $\nu=4$ (dotted), $\nu=5$ (dashed) and $\nu=6$ (long-dashed)

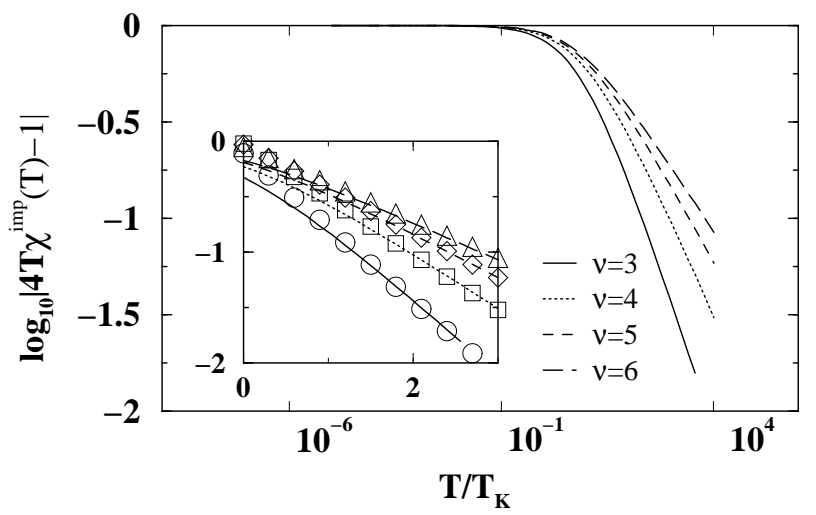

FIG. 4. The susceptibility at high temperatures, $T \gg T_{K}$, for anisotropies $\mu / \pi=1 / \nu$ and $\nu=3,4,5,6$. The impurity susceptibility at $T \gg T_{K}$ has the form $\chi^{\mathrm{imp}}(T)=\frac{1}{T}\left(1 / 4-B\left(T_{K} / T\right)^{2 \mu / \pi}\right)$, i.e. the corrections to the free behavior are power laws with exponents $2 \mu / \pi=2 / 3,1 / 2,2 / 5,1 / 3$ for $\nu=3,4,5,6$. This is illustrated in the inset, which shows $\log 4\left(T \chi^{\mathrm{imp}}(T)-1\right)$ versus $\log \left(T / T_{K}\right)$. Straight lines with slopes $-2 \mu / \pi=-1 / 3,-2 / 5,-1 / 2,-2 / 3$ for $\nu=3,4,5,6$ are indicated by symbols and are well reproduced by the numerical results.

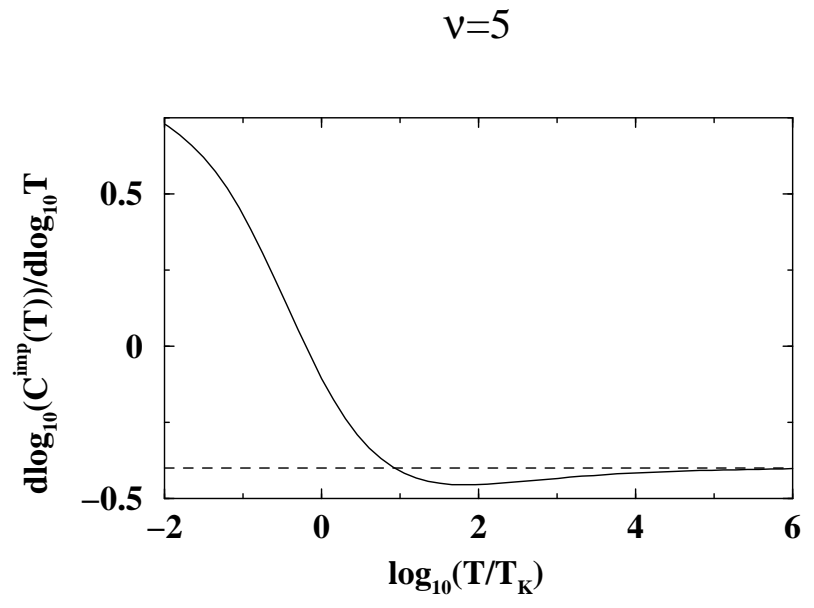

FIG. 5. The specific heat at high temperatures, $T \gg T_{K}$, shows power law behavior $C^{\mathrm{imp}}(T) \sim\left(T_{K} / T\right)^{2 \mu / \pi}$ with $\mu / \pi=1 / \nu$. The logarithmic derivative of this, $d \log C^{\mathrm{imp}}(T) / d \log T$, (solid line) approaches $-2 \mu / \pi$ at $T \gg T_{K}$ and is shown here for $\nu=5 \quad(\mu / \pi=1 / 5)$. It is seen to approach $-2 \mu / \pi=-2 / 5$ (dashed line) at high temperatures.

that, similar to the case dissipative two state systems, 12 it has power-law corrections in its thermodynamics at high temperatures $\left(T \gg T_{K}\right)$. The exponents are uniquely related to the anisotropy parameter $\mu$ (cf. Eq. 22). This is in contrast to the corresponding isotropic models which have logarithmic corrections at high temperatures. Fig. A and Fig. 5 show this for the susceptibility and specific heat, respectively.

Fig. 6 shows the remarkable magnetic field dependence of the local Wilson ratio, $R^{\mathrm{imp}}(h)$. This magnetic field dependence is consistent with the result for the isotropic case $\mu \rightarrow 0.32$ It is quite unexpected for the following reasons. First, the corresponding local Wilson ratio for the $f=1$ case discussed in Refs. [12,11,33]) is independent of the magnetic field and is given by

$$
R^{\mathrm{imp}, \mathrm{f}=1}=\frac{2}{\alpha_{1}}=\frac{2}{(1-\mu / \pi)} .
$$

It depends only on the anisotropy $\mu$ (that corresponds to the dissipation strength $\alpha_{1}$ in the equivalent dissipative two-state system 11 ). In contrast, for the present case $(f=2)$, the local Wilson ratio depends both on the anisotropy $\mu$ and on the magnetic field $h$. Thus, even though the regime $T \ll T_{\mathrm{FL}}$ describes a Fermi liquid, in the sense of Eq.29, the Fermi liquid state appears different to that for the $f=1$ case. We note that the local Wilson ratio for $f=2$ deviates increasingly from the usual $f=1$ Fermi-liquid Wilson ratio with decreasing $h$, i.e., as the range over which non-Fermi liquid behavior dominates increases. The numerical results for $R^{\mathrm{imp}}(h)$ in Fig. 6 indicate that $R^{\mathrm{imp}}(h)$ vanishes as $h \rightarrow 0$. This result is consistent with the numerical analysis of the 


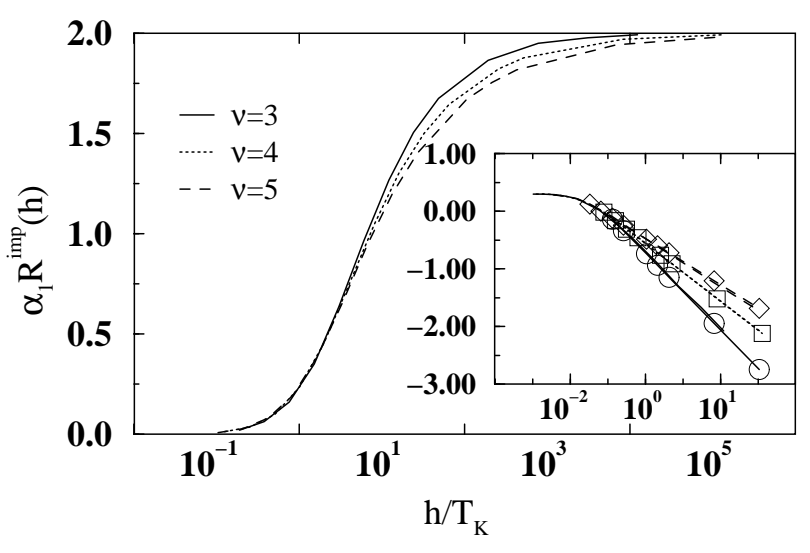

FIG. 6. The magnetic field $(h)$ dependence of the (local) Wilson ratio, $R^{\mathrm{imp}}(h)$ for different anisotropies $\mu / \pi=1 / \nu$ $\left(\nu=3\right.$ (solid), $\nu=4$ (dotted), $\nu=5$ (dashed)). At $h \gg T_{K}$, $\alpha_{1} R^{\mathrm{imp}}(h)=2-a\left(T_{K} / h\right)^{2 \mu / \pi}$. This is illustrated in the inset, which shows $\log \left(2-R^{\mathrm{imp}}(h)\right)$ versus $\log \left(h / T_{K}\right)$. Straight lines with slopes $-2 \mu / \pi=-2 / 3,-1 / 2,-2 / 5$, for $\nu=3,4,5$, are indicated by symbols and are well reproduced by the numerical results.

$h \rightarrow 0$ estimates of the susceptibility and specific heat in Eq. 28. In contrast, from Eq. 26 $\tilde{R}^{\mathrm{imp}}(T \rightarrow 0, h=0)$ is finite, so we see again that the two limits $T \rightarrow 0, h=0$ and $T=0, h \rightarrow 0$ cannot be exchanged. The inset to Fig. 6 shows that $R^{\text {imp }}(h)$ exhibits power law behavior at large magnetic fields. For $h \gg T_{K}$ we find

$$
\alpha_{1} R^{\mathrm{imp}}=2-a\left(T_{K} / h\right)^{2 \mu / \pi} .
$$

Finally, for completeness, we show in Fig. 7 the impurity magnetization (or polarizability) in the presence of a local field, $M_{z}(h)=\left\langle S_{0}^{z}\right\rangle(h)$. For each anisotropy, $\mu$, $M_{z}(h)$ is a universal function of $h / T_{K}$ (the same holds for $\left.R^{\mathrm{imp}}(h)\right)$. The approach of $M_{z}(h)$ to the free value for $h \gg T_{K}$ depends on anisotropy and is found numerically to behave like

$$
M_{z}(h)=1 / 2-b\left(T_{K} / h\right)^{2 \mu / \pi} .
$$

We expect that in the isotropic limit $\mu \rightarrow 0$ the above power laws will give way to logarithmic corrections in the same way that the power law corrections to the high temperature thermodynamics gave rise to logarithmic corrections in this limit.

\section{CONCLUSIONS}

In summary, we presented a detailed analysis of the thermodynamics of spin-anisotropic 2-channel Kondo model by using the Bethe Ansatz technique combined with bosonization and renormalization group arguments, and discussed quantitatively the role of the anisotropy and the magnetic field.

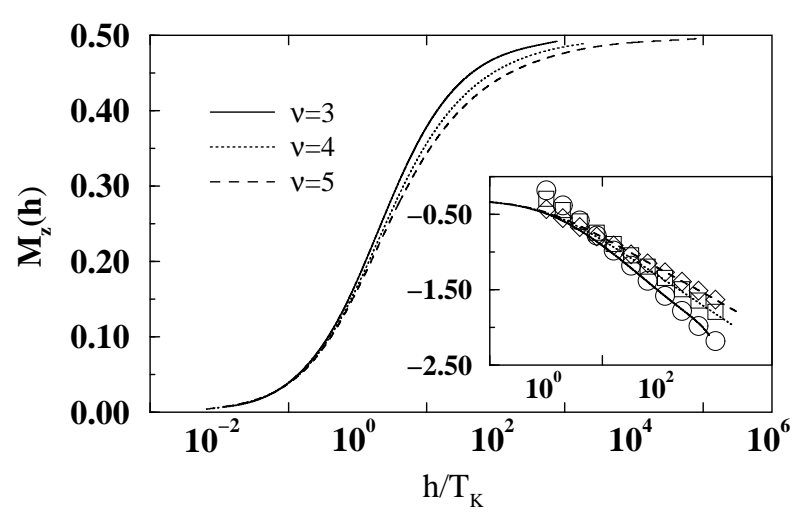

FIG. 7. The magnetic field $(h)$ dependence of the impurity magnetization, $M_{z}(h, T=0)$, for different anisotropies $\mu / \pi=1 / \nu(\nu=3$ (solid), $\nu=4$ (dotted), $\nu=5$ (dashed)). At $h \gg T_{K}, M_{z}(h)=1 / 2-b\left(T_{K} / h\right)^{2 \mu / \pi}$. This is illustrated in the inset, which shows $\log \left(1 / 2-M_{z}(h)\right)$ versus $\log \left(h / T_{K}\right)$. Straight lines with slopes $-2 \mu / \pi=-2 / 3,-1 / 2,-2 / 5$, for $\nu=3,4,5$, are indicated by symbols and are well reproduced by the numerical results.

We showed that at high temperatures the thermodynamics is very different from that of the isotropic model: The local impurity susceptibility is essentially free impurity-like, however, the coefficient of the global susceptibility is non-universal, and is related to the phase shift $\delta$ generated by the coupling $J_{z}$. In particular, the global susceptibility vanishes at the Emery-Kivelson line, $\delta=\pi / 2 f$. More interestingly, the impurity specific heat (and the corrections to the susceptibility about the free behavior) exhibits a power law behavior at high temperature

$$
C^{\mathrm{imp}}\left(T \gg T_{K}, h\right) \sim\left(\frac{T_{K}}{T}\right)^{2 \mu / \pi}
$$

The anomalous exponent $\mu$ is the anisotropy parameter in the Bethe Ansatz. We have shown that $\mu / \pi$ is just the anomalous scaling exponent of the spin flip term $J_{\perp}$ at high temperature and that if is related to the phase shifts generated by $J_{z}$ through 27

$$
\frac{\mu}{\pi}=4 \frac{\delta}{\pi}-4 f \frac{\delta^{2}}{\pi^{2}}
$$

On general scaling arguments, 27 a similar power-law dependence is expected to appear in the impurity resistivity. For $f=2$ we find

$$
\rho^{\mathrm{imp}}\left(T \gg T_{K}\right) \sim\left(\frac{T_{K}}{T}\right)^{2 \mu / \pi}
$$

rather then a simple logarithmic scaling.

For $h=0$, and for temperatures below the Kondo temperature, the thermodynamics is governed by the 
isotropic 2CK fixed point and most of the thermodynamic properties resemble very much those of the fully isotropic model, even for strong anisotropies.

For finite $h$ we showed that the non-Fermi liquid behavior found for $h=0$ persists for an intermediate region of temperatures $T_{\mathrm{FL}}<T<T_{K}$, provided $h<T_{K}$ so that the new scale $T_{\mathrm{F}} \sim h^{2} / T_{K}$ is well below $T_{K}$, just as in the isotropic case 32 We also showed that the Fermi liquid behavior below $T_{\mathrm{FL}}$ is unusual in that the Wilson ratio, $R^{\mathrm{imp}}(h)$, depended very sensitively on the magnetic field $h$. (in contrast to the $f=1$ case, which is completely independent of $h$ ) and we calculated the detailed dependence of this quantity for several anisotropies.

While here we focused our attention to specific values of the anisotropy, our calculations can be easily generalized to other anisotropy values and serve as a basis for any interpolation necessary for cases where there is wide distribution of anisotropies present.

The authors are grateful to A.M. Tsvelik and A. Zawadowski for useful discussions. This research has been supported by the Hungarian Grants OTKA T026327, OTKA F030041, and OTKA T029813, and NSF grant No. DMR 99-81283. The hospitality of the Institut-Laue Langevin during a visit (GZ) is gratefully .

\section{APPENDIX A: DERIVATION OF EQ. (17)}

To prove Eq. (17) let us consider the limit $J_{\perp} \rightarrow 0$ of Eq. (1D). In this case the interaction part of the Hamiltonian becomes:

$$
H_{\mathrm{int}}=\frac{J_{z}}{2} \sum_{j=1}^{f}\left(\psi_{\uparrow, j}^{\dagger}(0) \psi_{\uparrow, j}(0)-\psi_{\downarrow, j}^{\dagger}(0) \psi_{\downarrow, j}(0)\right) S_{0}^{z},
$$

where the electronic field operator $\psi_{\alpha, j}^{\dagger}$ creates chiral (right-moving) electron with spin $\alpha=\{+,-\}=\{\uparrow, \downarrow\}$ and channel index $j=\{1, . ., f\}$. Thus the interaction simply produces a spin-dependent potential scattering, and gives rise to a phase shift $\delta$

$$
\psi_{\alpha, j}\left(x=0^{+}\right)=\psi_{\alpha, j}\left(x=0^{-}\right) e^{-4 i \alpha \delta S_{0}^{z}} .
$$

In general, the connection between $J_{z}$ and $\delta$ depends on the particular cutoff scheme used except for the small coupling limit, $J_{z} \ll 1$. To be specific, here we shall use Abelian bosonization on a system ff finite size $L$ and the cutoff-scheme associated with it 30 In the bosonization procedure we rewrite the Hamiltonian as $\left(g=g^{\prime}=1\right)$ :

$$
\begin{aligned}
H & =\sum_{\alpha, j} \int \frac{d x}{4 \pi}\left(\partial_{x} \Phi_{\alpha, j}\right)^{2}+\frac{2 \pi}{L} \frac{1}{2} \sum_{j, \alpha} N_{\alpha, j}^{2} \\
& +\frac{J_{z}}{2} S_{0}^{z} \sum_{\alpha, j}\left(\frac{\alpha}{2 \pi} \partial_{x} \Phi_{\alpha, j}+\frac{1}{L} \alpha N_{\alpha, j}\right) \\
& +h\left(\sum_{\alpha, j} \frac{\alpha}{2} N_{\alpha, j}+S_{0}^{z}\right),
\end{aligned}
$$

where the external field $h$ couples to the total pseudospin of the system, $N_{\alpha, j}$ denotes the total number of electrons with respect to the ground state with spin $\alpha$ in channel $j$, and the free bosonic fields satisfy:

$$
\left[\partial_{x} \Phi_{\alpha, j}(x), \Phi_{\alpha^{\prime}, j^{\prime}}\left(x^{\prime}\right)\right]=-i 2 \pi \delta_{j j^{\prime}} \delta_{\alpha \alpha^{\prime}} \delta\left(x-x^{\prime}\right) .
$$

The original fermion fields can be represented as

$$
\psi_{\alpha, j}(x)=\frac{1}{\sqrt{a}} F_{\alpha, j} e^{-i \Phi_{\alpha, j}(x)},
$$

where $F_{\alpha, j}$ denotes the Klein factor, and $a$ is a small distance cut-off of the order of the lattice spacing.

The phase shift can be most easily calculated by introducing charge and spin fields and quantum numbers:

$$
\begin{aligned}
& \left(\begin{array}{l}
\Phi_{c, j} \\
\Phi_{s, j}
\end{array}\right) \equiv \frac{1}{\sqrt{2}}\left(\begin{array}{rr}
1 & 1 \\
1 & -1
\end{array}\right)\left(\begin{array}{l}
\Phi_{\uparrow, j} \\
\Phi_{\downarrow, j}
\end{array}\right), \\
& N_{c, j} \equiv N_{\uparrow, j}+N_{\downarrow, j}, \\
& N_{s, j} \equiv \frac{1}{2}\left(N_{\uparrow, j}-N_{\downarrow, j}\right),
\end{aligned}
$$

and performing a unitary transformation on the Hamiltonian by $U=e^{i \sum_{j} J_{z} \Phi_{s, j}(0) S_{0}^{z} /(2 \pi \sqrt{2})}$, resulting in

$$
\partial_{x} \Phi_{s, j}(x) \rightarrow \partial_{x} \Phi_{s, j}(x)-\frac{J_{z}}{\sqrt{2}} S_{0}^{z} \delta(x),
$$

and the 'noninteracting' Hamiltonian:

$$
\begin{aligned}
H & =H_{0}+H_{s} \\
H_{0} & =\sum_{j=1}^{f} \sum_{\mu=c, s} \int \frac{d x}{4 \pi}\left(\partial_{x} \Phi_{\mu, j}\right)^{2}+\frac{2 \pi}{L} \frac{1}{4} \sum_{j} N_{c, j}^{2}, \\
H_{s} & =h S_{0}^{z}+\sum_{j}\left(h N_{s, j}+\frac{2 \pi}{L} N_{s, j}^{2}+J_{z} \frac{N_{s, j}}{L} S_{0}^{z}\right) .
\end{aligned}
$$

From Eqs. (A9), ( $\mathrm{A6}),(\mathrm{A} 5)$, and ( $\mathrm{A2}$ ) immediately follows that in the bosonization cut-off scheme simply

$$
\delta=\frac{J_{z}}{8} .
$$

To prove Eq. (17) we observe that the external field only appears in (A12). Therefore the partition function factorizes as

$$
\begin{aligned}
Z(\beta) & =Z_{0}(\beta) \times Z_{s}(\beta, h), \\
Z_{s}(\beta, h) & =\sum_{N_{s}} \sum_{S_{0}^{z}= \pm 1 / 2} e^{-\beta H_{s}} .
\end{aligned}
$$

It is easy to evaluate the sum above in the $L \rightarrow \infty$ limit giving:

$$
Z_{s} \sim e^{\beta f \frac{L}{2 \pi} \frac{1}{4} h^{2}} \times \cosh \left[\frac{\beta h}{2}\left(1-f \frac{J_{z}}{4 \pi}\right)\right] .
$$

The first term just generates the Pauli susceptibility of a free electron gas, while the second corresponds to a free 
spin coupled to a renormalized magnetic field and gives a Curie susceptibility:

$$
\chi_{\text {glob }}^{\mathrm{TLS}}=\frac{\left(1-f J_{z} / 4 \pi\right)^{2}}{4 T} .
$$

Together with Eq. (A13), this yields Eq. (17).

As a further test, one can compare this result with the exact relation, Eq. (16) in the small compling limit. Within the Bethe Ansatz cutoff scheme $22 \cos (\mu)=$ $\cos \left(J_{z} / 2\right) / \cos \left(J_{\perp} / 2\right)$, which in the appropriate small coupling limit gives $\mu=J_{z} / 2+\mathcal{O}\left(J_{z}^{2}\right)=4 \delta+\mathcal{O}\left(\delta^{2}\right)$. Substituting this expression into Eq. (16) we indeed recover the exact relation Eq. (17) in linear order in $\delta$.

${ }^{1}$ For a review see D.L. Cox and A. Zawadowski, Adv. Phys. 47, 599 (1998).

${ }^{2}$ K. Vladár and A. Zawadowski, Phys. Rev. B 28, 1564, 1582, 1596 (1983).

${ }^{3}$ I.L. Aleiner, B.L. Altshuler, Y.M. Galperin, T.A. Shutenko, Phys. Rev. Lett. 86, 2629 (2001).

${ }^{4}$ I. Smolyarenko and N.S. Wingreen, Phys. Rev. B 60, 9675 (1999).

${ }^{5}$ D.C. Ralph, A.W.W. Ludwig, Jan von Delft, and R.A. Buhrman, Phys. Rev. Lett. 72, 1064 (1994);

${ }^{6}$ S.K. Upadhyay, R.N. Louie, R.A. Buhrman, Phys. Rev. B 56, 12033 (1997).

${ }^{7}$ G. Zaránd, Jan von Delft, and A. Zawadowski, Phys. Rev. Lett. 80, 1353 (1998).

${ }^{8}$ L. I. Glazman and K. A. Matveev, Sov. Phys. JETP 71, 1031 (1990).

${ }^{9}$ G. Zaránd, G.T. Zimányi, and F. Wilhelm, Phys. Rev. B 628137 (2000).

${ }^{10}$ D. Berman, N.B. Zhetinev, and R.C. Ashoori, Phys. Rev. Lett. 82, 161 (1999).

11 T. A. Costi and G. Zaránd, Phys. Rev. B 59, 12398 (1999).

12 A. M. Tsvelik and P. B. Wiegmann, Adv. Phys. 32, 453 (1983).

${ }^{13}$ I. Affleck and A. W. W. Ludwig, Phys. Rev. B 48, 7297 (1993).

14 J.H. Lowenstein, Phys. Rev. 29, 4120 (1984).

${ }^{15}$ P. B. Vigmann and A. M. Finkel'stěin, Sov. Phys. JETP 48,102 (1978).

${ }^{16}$ N. Andrei and A. Jerez, Phys. Rev. Lett. 74, 4507 (1995).

${ }^{17}$ N. Andrei and C. Destri, Phys. Rev. Lett. 52, 364 (1984).

${ }^{18}$ M. Takahashi and M. Suzuki, Prog. Theor. Phys. 48, 2187 (1972).

19 A. M. Tsvelik, Phys. Rev. B 52, 4366-4370 (1995).

${ }^{20}$ V. J. Emery and S. A. Kivelson, in Fundamental Problems in Statistical Mechanics VIII, edited by H. van Beijeren and M. H. Ernst, Elsevier, Amsterdam, (1994).

${ }^{21}$ N. Andrei, in Series on Modern Condensed Matter Physics - Vol. 6, 458-551, World Scientific, Lecture Notes of ICTP Summer Course, September 1992. Editors: S. Lundquist, G. Morandi and Yu Lu.
22 To obtain the prefactor in Eq. (5) one has to study the Toulouse limit of Ref. [19].

${ }^{23} c_{\text {bulk }}$ in Eq. (15) denotes the total specific heat of the electron gas, which cannot be obtained from the fused BA equations, that capture genly contributions from the $S U(2)_{f}$ spin sector (see e.g. Ref 34 ).

${ }^{24}$ H.-B. Pang and D. L. Cox, Phys. Rev. B 44, 9454 (1991).

${ }^{25}$ G. Zaránd, and J. von Delft, Phys. Rev. B 61, 6918 (2000).

${ }^{26}$ J. W. Ye, Phys. Rev. Lett. 77, 3224 (1996).

${ }^{27}$ K. Vladár, A. Zawadowski and G. T. Zimànyi, Phys. Rev. B 37, 2001, 2015 (1988).

${ }^{28}$ V. J. Emery and S. Kivelson, Phys. Rev. B 46, 10812 (1992); A. M. Sengupta and A. Georges, Phys. Rev. B 49, 10020 (1994).

${ }^{29} \mathrm{By} \mathrm{Eq.} \mathrm{(18)} \mathrm{the} \mathrm{rescaling} \mathrm{of} g$ exactly compensates the rescaling of $h$, and therefore the BA equations with $g=1$ and $g^{\prime}=1$ determine directly the impurity free energy.

${ }^{30}$ Jan bosonization.

31 Note, however, that the corresponding global impurity Wilson ratio for the $f=1$ case is independent of both the magnetic field ant the anisotropy, and takes the usual value $R_{\text {glob }}^{\text {imp } \mathrm{f}=1}=2211$.

32 P. D. Sacramento and P. Schlottman, Phys. Rev. B 43, 13294 (1991).

${ }^{33}$ M. Sassetti and U. Weiss, Phys. Rev. Lett. 65, 2262 (1990)

${ }^{34}$ N. Andrei, M. Douglas, and A. Jerez, Phys. Rev. B 58, 7619 (1998). 